\title{
A web based relational database management system for filariasis control
}

\author{
Upadhyayula Suryanarayana Murty ${ }^{1 *}$, Duvvuri Venkata Rama Satya Kumar ${ }^{1}$, Kumaraswamy Sriram ${ }^{1}$, Kadiri \\ Madhusudhan Rao ${ }^{1}$, Chakravarthula Hayageeva Narasimha Venakata Bhattacharyulu', Bhoopathi Praveen ${ }^{1}$ and \\ Amirapu Radha Krishna ${ }^{2}$ \\ ${ }^{1}$ Bioinformatics Group, Biology Division, Indian Institute of Chemical Technology (CSIR), \\ Hyderabad - 500 007, Andhra Pradesh, India; ${ }^{2}$ Computer Division, Indian Institute of Chemical Technology (CSIR), Hyderabad \\ - 500 007, Andhra Pradesh, India; \\ Upadhyayula Suryanarayana Murty* - Email: murty_usn@yahoo.com; * Corresponding author \\ received April 25, 2005; revised April 29, 2005; accepted April 30, 2005; published online April 30, 2005
}

\begin{abstract}
:
The present study describes a RDBMS (relational database management system) for the effective management of Filariasis, a vector borne disease. Filariasis infects 120 million people from 83 countries. The possible re-emergence of the disease and the complexity of existing control programs warrant the development of new strategies. A database containing comprehensive data associated with filariasis finds utility in disease control. We have developed a database containing information on the socioeconomic status of patients, mosquito collection procedures, mosquito dissection data, filariasis survey report and mass blood data. The database can be searched using a user friendly web interface.
\end{abstract}

Keywords: Filariasis; web-based; RDBMS; control measures; disease management

Availability: http://www.webfil.org (login and password can be obtained from the authors)

\section{Background:}

Filariasis is a serious public health problem. [1] It is one of the leading causes of disability in 48 million people from the Indian sub-continent. The annual economic loss due to filariasis burden is huge. [2, 3] The disease is caused by nematode worms Wuchereria bancrofti, Brugia malayi and Brugia timori transmitted by female mosquitoes Culex, Anopheles, Mansoniods and others. [3] The NFCP (National Filaria Control Programme) was launched in India during 1955. However, the NFGP showed limited progress due to lack of skilled manpower, inadequate funding, improper estimation of vector/disease transmission rates, improper implementation of control programmes and ineffective communication between the field workers and health administrators. [4, 5] Hence, a database is necessary to identify parameters representing the spread of the disease. This will help in the design of effective control strategies. Here, we describe a database containing information on filariasis.

\section{Methodology:}

Dataset:

The epidemiology, entomology and socio-economic data associated with filariasis were collected from 45 rural areas in the Indian state of Andhra Pradesh during 1998 to 2001.

\section{Database Interface:}

The database can be accessed using login and password obtained from the authors. The interface helps to search the database based on epidemiological, entomological and socioeconomical parameters (Figure 1A).

\section{Database design:}

The database consists of two suites, namely, (1) data entry suite, and (2) report generation suite. The data entry suite contains ADD, MODIFY, DELETE and SEARCH options for effective and easy update.

\section{Data entry suite:}

Data entry is feasible for socio-economic data (serial number, date, village, taluk, district, unit name, sex, age, affected organ, occupation, family background, filariasis awareness, mosquito avoidance, water supply, structure of house, presence of animals, habitat, number of earning members of the family, average income and education status of parents), mosquito collection data (adult mosquito count, species name, type of catching stations (random/fixed), total collection, time spent, male mosquitoes number, females mosquitoes number, and density per 10 man hour (10MHD)), mosquito dissection data (infection rate and infectivity rate), filariasis survey (disease rate) and mass blood survey (micro-filarial rate in night blood).

10MHD is calculated as (total female mosquitoes collected/time spent)*10. The infection rate and infectivity rate is defined as (female mosquitoes (Culex quinquefasciatus) with all stages of larvae/total mosquitoes dissected)*100 and (female mosquitoes (Culex quinquefasciatus) with III stage of larvae/total mosquitoes dissected)*100, respectively. Disease rate is estimated as (total diseased/total sampled)*100. Micro-filarial rate is defined as (positive blood samples/total blood samples collected)*100. 


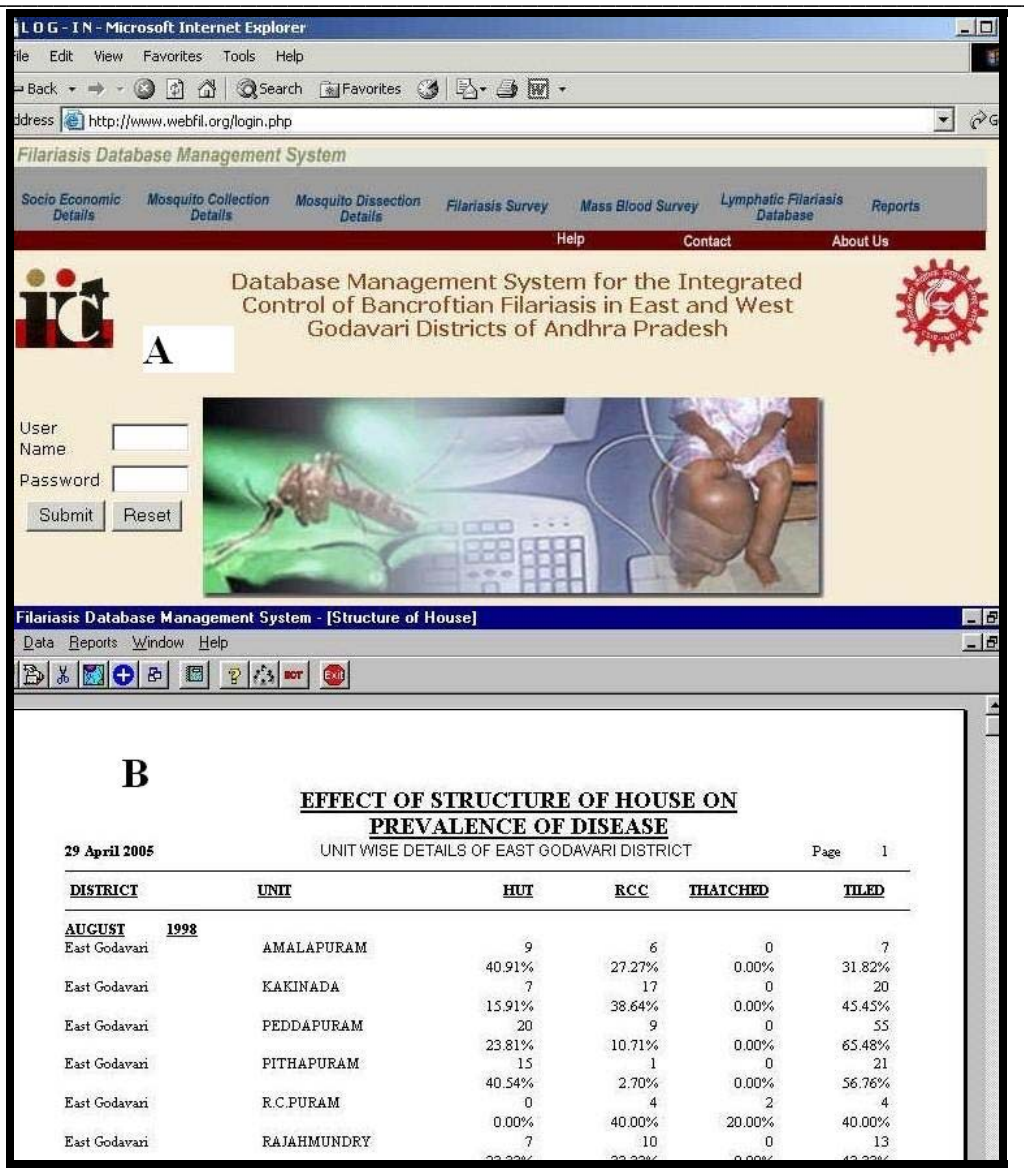

Figure 1: A web database for filariasis management. (A) web interface, (B) report generated in the database

\section{Report generation suite:}

The database facilitates the generation of a report consisting of several information in the database according to the input parameters selected by the user (Figure 1B).

\section{Utility:}

The RDBMS system for filariasis is critical for the understanding of vector dynamics towards disease management. This will help to develop effective control programs. We propose to develop similar RDBMS system for diseases like Malaria, Japanese encephalitis, Dengue and others.

\section{Acknowledgement:}

The database was developed and managed by Indian Institute of Chemical Technology (CSIR), Hyderabad, India. This is funded by the Ministry of Communication and Information Technology, Government of India, New Delhi.

\section{References:}

[1] D. M. Wayne, Int J Parasitol., 32:947 (2002) [PMID: 12076624]

[2] E. A. Ottesen, et al, Bull.Wld.Hlth.Org., 75:491 (1997) [PMID: 9509621]

[3] K. D. Ramaiah, et al., Parasitol. Today, 16:251 (2000) [PMID: 10827432]

[4] K. D. Ramaiah, et al., Indian J Med Res., 89:184 (1989) [PMID: 2674003]

[5] U. Suryanarayana Murty, et al., South-East Asian J Trop Med. Pub. Hlt., 33:702 (2002) [PMID: 127572131]

Edited by M. Ravichandran

Citation: Murty et al., Bioinformation 1(1): 19-20 (2005)

License statement: This is an open-access article, which permits unrestricted use, distribution, and reproduction in any medium, for non-commercial purposes, provided the original author and source are credited. 